\title{
EDITORIAL
}

\section{KMC Practices During COVID-19 Pandemic}

\author{
Md. Mahbubul Hoque
}

Preterm and low birth weight newborns are highly vulnerable population for whom high quality care from health services is imperative. More than $80 \%$ of the world's annual neonatal deaths (2.5 million) occur in babies with a low birth weight (LBW, $<2500$ g), among which two-thirds are preterm $(<37$ completed weeks of gestation) and one third are small-for-gestational-age. ${ }^{1-3}$ To achieve NMR target of SDG it is mediatory to reduce death from prematurity as the complications of prematurity are the leading cause of death in neonates and children aged $<5$ years. ${ }^{4}$

Kangaroo mother care (KMC) involves continuous skin-to-skin contact between a newborn and a caregiver (usually the mother). It is a model of care that is an alternative to the incubator for preterm newborns. The World Health Organization (WHO) has recommended this type of care in both developed and developing countries as soon as the premature neonate is clinically stabilized. ${ }^{5}$ Kangaroo mother care includes early and continuous skin-to-skin contact, breastfeeding, early discharge from the health-care facility and supportive care. The clinical efficacy and health benefits of kangaroo mother care have been demonstrated in multiple settings. In low birth weight newborns $(<2000 \mathrm{~g})$ who are clinically stable, kangaroo mother care reduces mortality and if widely applied could reduce deaths in preterm newborns. ${ }^{6,7}$

However, in spite of the evidence, KMC practices have been interrupted by the COVID-19 pandemic. Owing to the high contagion and fatality rate of the virus and the WHO declaration of COVID-19 as a pandemic, routine medical care has been impacted and thus the rate of KMC has suffered consequently. The COVID-19 pandemic is disrupting facility-based care. Further, mothers in facility settings are more frequently being separated from their newborns.

As clinical evidence shows KMC is highly effective in the management of preterm baby - development partners, professional bodies and countries set together several times and discussed how to sustain KMC practices during the COVID-19 pandemic. Different countries made their own guidelines on the management issue of neonate and child during COVID-19 pandemic. Initially there were conflicting global guidelines on mother-newborn care during the pandemic, particularly regarding skin-to-skin contact, and few such guidelines specifically for low- and middle-income countries (LMIC). A systematic review of 20 clinical guidelines from 17 countries found that one third of them recommended mother-newborn separation. ${ }^{8}$ However, Bangladesh continues to promote KMC amidst COVID-19 pandemic to prevent prematurity related complications and deaths, which was possible by combined efforts from DGHS of GoB, WHO, UNICEF, USAID, Save the Children and professional bodies' like Bangladesh Neonatal Forum (BNF), Bangladesh Paediatric Association (BPA), Bangladesh Perinatal Society (BPS).

The WHO recommended that mothers and newborns should not be separated. The dyads should enable the practice of KMC even in cases of suspected or confirmed COVID-19 by using personal protective equipment and the disinfection of used surfaces. ${ }^{9}$

A newly published study using the British Paediatric Surveillance Unit reported 66 cases of SARS-CoV-2 among neonates receiving inpatient care in the UK between March and April 2020, of whom 17(26\%) were born to mothers with perinatal infection. Seven of these 17 neonates became infected despite being separated from their mother immediately after birth, supporting WHO and other development partners and professional bodies guidance to keep mother and baby together even when maternal COVID-19 is suspected or confirmed. ${ }^{10}$

A recent study showing the comparative risk analysis of maximum neonatal lives saved by $\mathrm{KMC}$, versus maximum lives lost due to COVID-19, and

Correspondence to: Prof. Md. Mahbubul Hoque, Professor, Department of Neonatal Medicine, Bangladesh Institute of Child Health and Dhaka Shishu (Children) Hospital. Cell: 01729290121, E-mail: mahbubulhoque2013@gmail.com 
incremental deaths caused by reduced KMC coverage in facilities. ${ }^{11}$ In this research they modeled two scenarios over 12 months. Scenario 1 compared the survival benefits of KMC with universal coverage (99\%) and mortality risk due to COVID-19. Scenario 2 estimated incremental deaths from reduced coverage and complete disruption of KMC. Projections were based on the most recent data for 127 LMICs ( 90\% of global births), with results aggregated into five regions. Hence, the benefit of $\mathrm{KMC}$ is 65 -fold higher than the mortality risk of COVID-19 and estimated $2 \cdot 3 \cdot 4 \cdot 6 \%$ increase in neonatal mortality across the 127 countries. ${ }^{11}$

Preterm newborns are at risk, especially in LMICs where the consequences of disruptions are substantial. As the survival benefit of KMC far outweighs the small risk of death due to COVID-19 we urge policymakers and healthcare professionals to protect services for preterm and as such consider $\mathrm{KMC}$ in the neonatal wards, with the use of all related precautions, even if the mother is SARS-CoV-2positive.

\section{References}

1. UN Inter-agency Group for Child Mortality Estimation. Levels \& trends in child mortality: report 2020. New York; 2020. [cited 2020 Oct 9]. Available from: https://data.unicef.org/resources/levels-andtrends-in-child-mortality/.

2. Lawn J, Blencowe H, Oza S, You D, Lee ACC, Waiswa P, et al. Every newborn: Progress, priorities, and potential beyond survival. Lancet 2014;384:189-205.

3. Blencowe H, Krasevec J, de Onis M, Black RE, Xiaoyi An X, Stevens GA, et al. National, regional, and worldwide estimates of low birthweight in 2015, with trends from 2000: A systematic analysis. Lancet Glob Health 2019;7:e849-60.

4. UN Inter-agency Group for Child Mortality Estimation. Country under-five, infant, child and neonatal mortality, 2017. New York; 2020. [cited
2020 Sep 18]. Available from: https:// childmortality.org/wp-content/uploads/2020/09/ UNIGME-2020-Country-Rates-Deaths-Underfive.xlsx.

5. World Health Organization. WHO recommendations on newborn health: Guidelines approved by the WHO Guidelines Review Committee. https://apps.who.int/ iris/bits. Updated May 2017. Accessed April 23, 2020.Google Scholar

6. Hoque MM, Jahan N, Rahman MM, Saha LC, Akhter RJ, Chowdhury MAKA. Effectiveness of KMC on success of breast feeding in preterm low birth weight neonate. Acad J Ped Neonatol 2017;3:70-73.

7. Ramanathan K, Paul VK, Deorari AK, Taneja U, George G. Kangaroo mother care in very low birth weight infants. Indian J Pediatr 2001;68:1019-23.

8. Yeo KT, Oei JL, De Luca D, Schmolzer GM, Guaran $\mathrm{R}$, Palasanthiran P, et al. Review of guidelines and recommendations from 17 countries highlights the challenges that clinicians face caring for neonates born to mothers with COVID-19. Acta Paediatr 2020;109:2192-207.

9. World Health Organization. Clinical management of severe acute respiratory infection (SARI) when COVID-19 disease is suspected. https://www.who.int/ publication. March 13, 2020. Accessed April 23, 2020. Google Scholar

10. Gale C, Quigley MA, Placzek A, Knight M, Ladhani S, Draper ES, et al. Characteristics and outcomes of neonatal SARS-CoV-2 infection in the UK: A prospective national cohort study using active surveillance. Lancet Child Adolesc Health 2020;5:P113-21.

11. Minckas N, Medvedev MM, Adejuyigbed EA, Brothertonc H, Chellanif H, Estifanosg AS, et al. Preterm care during the COVID-19 pandemic: A comparative risk analysis of neonatal deaths averted by kangaroo mother care versus mortality due to SARS-CoV-2 infection, E Clinical Medicine (2021), https://doi.org/10.1016/j. eclinm.2021.10073. 